PROCEEDINGS OF THE

AMERICAN MATHEMATICAL SOCIETY

Volume 126, Number 8, August 1998, Pages 2221-2228

S 0002-9939(98)04479-7

\title{
AUTOMORPHIC-DIFFERENTIAL IDENTITIES AND ACTIONS OF POINTED COALGEBRAS ON RINGS
}

\author{
TADASHI YANAI
}

(Communicated by Ken Goodearl)

\begin{abstract}
In this paper, we prove the following two results which generalize the theorem concerning automorphic-differential endomorphisms asserted by J. Bergen. Let $R$ be a ring, $R_{\mathcal{F}}$ its left Martindale quotient ring and $\mathfrak{A}$ a right ideal of $R$ having no nonzero left annihilator. (1) Let $C$ be a pointed coalgebra which measures $R$ such that the group-like elements of $C$ act as automorphisms of $R$. If $R$ is prime and $\xi \cdot \mathfrak{A}=0$ for $\xi \in R \# C$, then $\xi \cdot R=0$. Furthermore, if the action of $C$ extends to $R_{\mathcal{F}}$ and if $\xi \in R_{\mathcal{F}} \# C$ such that $\xi \cdot \mathfrak{A}=0$, then $\xi \cdot R_{\mathcal{F}}=0$. (2) Let $f$ be an endomorphism of $R_{\mathcal{F}}$ given as a sum of composition maps of left multiplications, right multiplications, automorphisms and skew-derivations. If $R$ is semiprime and $f(\mathfrak{A})=0$, then $f(R)=0$.
\end{abstract}

\section{INTRODUCTION}

The motivation of this note is the following problem in [B, Question 4], posed by J. Bergen.

Question. Suppose $R$ is a ring, $H$ a Hopf algebra acting on $R$, and let $f \in R \# H$. If $R$ is special, such as being prime or a domain, and if $\lambda \neq 0$ is a special subset of $R$, such as being an ideal or a one-sided ideal, must $f \cdot R=0$ whenever $f \cdot \lambda=0$ ?

This question is mentioned as a consequence of the theorem concerning a special mapping, called an automorphic-differential endomorphism, in prime rings. Let $\operatorname{End}(R,+)$ be the ring consisting of all additive endomorphisms of a ring $R$. For $a \in R, T_{a}$ and $L_{a}$ represent the right and left multiplication maps of $R$ respectively. Let $\mathcal{A}$ be the subring of $\operatorname{End}(R,+)$ generated by $\left\{T_{a}, L_{a} \mid a \in R\right\}$, all automorphisms and derivations of $R$. An element of $\mathcal{A}$ is called an automorphic-differential endomorphism of $R$. For a subset $A \subseteq R$, we set $\ell(A)=\{a \in R \mid a A=0\}$ and $r(A)=\{a \in R \mid A a=0\}$. Bergen proved the following theorem.

Theorem A ([B, Theorem 1$])$. Let $\mathfrak{A}$ be a right ideal of a prime ring $R$ with $\ell(\mathfrak{A})=0$ and $f$ an automorphic-differential endomorphism of $R$. If $f(\mathfrak{A})=0$, then we have $f(R)=0$.

Our first aim is to extend this fact to the action of a pointed coalgebra on prime rings. For this purpose, we use the Taft-Wilson theorem, which determines the structure of the coradical filtration of a pointed coalgebra.

Received by the editors May 31, 1996 and, in revised form, October 24, 1996 and January 24, 1997.

1991 Mathematics Subject Classification. Primary 16W20, 16W25, 16W30. 
Theorem 2. Let $R$ be a prime algebra and $C$ a pointed coalgebra which measures $R$ such that the group-like elements of $C$ act as automorphisms of $R$. If $\xi \cdot \mathfrak{A}=0$ for $\xi \in R \# C$ and a right ideal $\mathfrak{A}$ of $R$ with $\ell(\mathfrak{A})=0$, then $\xi \cdot R=0$. Furthermore, if the action of $C$ extends to $R_{\mathcal{F}}$ and if $\xi \in R_{\mathcal{F}} \# C$ such that $\xi \cdot \mathfrak{A}=0$, then $\xi \cdot R_{\mathcal{F}}=0$.

In [B, Question 1], Bergen also asked whether Theorem A holds even if $R$ is semiprime, and in $[\mathrm{O}], \mathrm{A}$. Ouarit gave an affirmative answer to this problem. We extend this result to an endomorphism with skew-derivations, using a different proof from $[\mathrm{O}]$, in Section 4.

Theorem 6. Let $R$ be a semiprime ring and $f$ an automorphic-skew-differential endomorphism. If $f(\mathfrak{A})=0$ for some right ideal $\mathfrak{A}$ with $\ell(\mathfrak{A})=0$, then $f(R)=0$.

\section{Martindale Quotient Rings and aCtions of COALGebras}

We recall some notions which we need in this paper. Let $R$ be an arbitrary ring and $\mathcal{F}$ the set of all ideals $I$ of $R$ with $r(I)=\ell(I)=0$. If $R$ is a prime ring, $\mathcal{F}$ is the set of all nonzero ideals; and if $R$ is semiprime, all essential ideals. We denote the left Martindale quotient ring of $R$ by $R_{\mathcal{F}}$, the symmetric quotient ring of $R$ by $Q$ and the extended centroid by $K$. (See $[\mathrm{K} 2, \S 1.4],[\mathrm{M} 2, \mathrm{p} .97]$ for detailed definitions.) If $R$ is semiprime, it is known that $R_{\mathcal{F}}$ and $Q$ are semiprime rings and $K$ is a von Neumann regular ring; and if $R$ is prime, $R_{\mathcal{F}}$ and $Q$ are prime rings and $K$ is a field.

We have the following facts on $R_{\mathcal{F}}$ and $Q$.

1. For any $q \in R_{\mathcal{F}}$, there exists $I \in \mathcal{F}$ with $I q \subseteq R$.

2. For any $q \in Q$, there exists $I \in \mathcal{F}$ with $q I \subseteq R$.

For nonempty subsets $A, B \subseteq R_{\mathcal{F}}$, we define $\ell_{A}(B)=\{a \in A \mid a B=0\}$. Throughout, $C$ represents a coalgebra over a field $\mathbf{k}$ with comultiplication $\Delta$ and counit $\varepsilon$. We use the sigma notation as follows : $\Delta(c)=\sum c_{1} \otimes c_{2}$ for $c \in C$. Let $R$ be a k-algebra. We say that $C$ measures $R$ if an action $C \otimes R \ni c \otimes r \mapsto c \cdot r \in R$ satisfies the following conditions:

(1) for any $a, b \in R$ and $c \in C, c \cdot(a b)=\sum\left(c_{1} \cdot a\right)\left(c_{2} \cdot b\right)$, and

(2) $c \cdot 1=\varepsilon(c) 1$ for any $c \in C$.

We can define an $(R, R)$-bimodule $R \# C$ as follows:

(1) $R \# C=R \otimes C$ as a $\mathbf{k}$-space,

(2) the element $a \otimes c$ is denoted by $a \# c$, and

(3) the left (right) action of $R$ is given by

$$
a^{\prime}(a \# c)=a^{\prime} a \# c \quad\left((a \# c) a^{\prime}=\sum a\left(c_{1} \cdot a^{\prime}\right) \# c_{2}\right)
$$

for $a, a^{\prime} \in R$ and $c \in C$.

For $\xi=\sum a_{i} \# c_{i} \in R \# C$ and $r \in R$, we define $\xi \cdot r=\sum a_{i}\left(c_{i} \cdot r\right)$. It is easy to show that for $\xi \in R \# C$ and $a, r \in R$, we have $(\xi a) \cdot r=\xi \cdot(a r)$ and $(a \xi) \cdot r=a(\xi \cdot r)$.

\section{ON PRIME RINGS WITH ACTIONS OF POINTED COALGEBRAS}

We recall the coradical filtration of a coalgebra. For a coalgebra $C$, the coradical $C_{0}$ is the sum of all simple subcoalgebras of $C$. For $n \geq 1$,

$$
C_{n}=\Delta^{-1}\left(C_{0} \otimes C+C_{n-1} \otimes C\right) \text {. }
$$


$C_{n}$ is a subcoalgebra of $C$ for all $n$, and $C=\bigcup_{n=0}^{\infty} C_{n}$. If $C$ is pointed, then $C_{0}=\mathbf{k} G$, where $G$ is the set of all group-like elements of $C$, i.e. $G=\{0 \neq \sigma \in C \mid \Delta(\sigma)=$ $\sigma \otimes \sigma\}$. For $\sigma, \tau \in G$, we set

(1) $X_{\sigma, \tau, n}=\left\{x \in C_{n} \mid \Delta(x) \in \sigma \otimes x+x \otimes \tau+C_{n-1} \otimes C_{n-1}\right\}(n \geq 1)$,

(2) if $\sigma=\tau$, then $X_{\sigma, \tau, 0}=\mathrm{k} \sigma$; otherwise $X_{\sigma, \tau, 0}=0$,

(3) $X_{\sigma, \tau}=\sum_{n \geq 0} X_{\sigma, \tau, n}$.

We state the Taft-Wilson theorem.

Proposition 1 ([TW], [Ma]). Let $C$ be a pointed coalgebra and $\left\{C_{n}\right\}_{n=0}^{\infty}$ its coradical filtration. Set $C_{-1}=0$. For each non-negative integer $n$, there exists a subspace $X_{n}$ of $C_{n}$ with $C_{n}=X_{n} \oplus C_{n-1}$ as a $\mathbf{k}$-space. Moreover, for the above $X_{\sigma, \tau, n}$

(1) $X_{n}=\sum_{\sigma, \tau} X_{\sigma, \tau, n}$, and

(2) $\Delta\left(X_{\sigma, \tau}\right) \subseteq \sigma \otimes X_{\sigma, \tau}+X_{\sigma, \tau} \otimes \tau+C_{n-1} \otimes C_{n-1}$.

We are ready to prove our first main theorem.

Theorem 2. Let $R$ be a prime algebra and $C$ a pointed coalgebra which measures $R$ such that the group-like elements of $C$ act as automorphisms of $R$. If $\xi \cdot \mathfrak{A}=0$ for $\xi \in R \# C$ and a right ideal $\mathfrak{A}$ of $R$ with $\ell(\mathfrak{A})=0$, then $\xi \cdot R=0$. Furthermore, if the action of $C$ extends to $R_{\mathcal{F}}$ and if $\xi \in R_{\mathcal{F}} \# C$ such that $\xi \cdot \mathfrak{A}=0$, then $\xi \cdot R_{\mathcal{F}}=0$.

Proof. Let $B$ denote either $R$ or $R_{\mathcal{F}}$, depending upon whether we are proving the first or second part of the theorem. In any case, we can assume that $C$ acts on $B$, $\xi \cdot \mathfrak{A}=0$, and we need to show that $\xi \cdot B=0$.

Suppose the contrary. This means that the set $D=\{\xi \in B \# C \mid \xi \cdot \mathfrak{A}=0$ and $\xi \cdot B \neq 0\}$ is not empty. Let $n$ be the smallest integer satisfying $D \cap\left(B \# C_{n}\right) \neq \emptyset$. From the Taft-Wilson theorem, we have a basis $\left\{x_{i}\right\}$ for $X_{n}$ satisfying $\Delta\left(x_{i}\right) \in$ $\sigma_{i} \otimes x_{i}+C \otimes C_{n-1}$ for $\sigma_{i} \in G$. An element of $D \cap\left(B \# C_{n}\right)$ can be written in the form $\sum a_{i} \# x_{i}+y$ for $a_{i} \in B$ and $y \in B \# C_{n-1}$. We take an element $\xi$ for which the number of terms $a_{i} \# x_{i}\left(a_{i} \neq 0\right)$ appearing in $\xi$ is least among all elements of $D \cap\left(B \# C_{n}\right)$. Now, set $\xi=\sum_{i=1}^{k} a_{i} \# x_{i}+y\left(0 \neq a_{i} \in B, y \in B \# C_{n-1}\right)$.

If $a_{1} \notin R$, multiplying by a suitable element of $I_{1} \in \mathcal{F}$ with $I_{1} a_{1} \subseteq R$, we may assume that $a_{1}$ is contained in $R$.

By assumption, the mapping $g_{1}: B \rightarrow B$ given by $q \mapsto \sigma_{1} \cdot q$ is an automorphism of $B$ with $g_{1}^{-1}(R) \subseteq R$.

There exists some $b \in B$ such that $\xi \cdot b \neq 0$. Let $I \neq 0$ be an ideal of $R$ such that $I a_{1} g_{1}(b) \subseteq R$. If we let $r \in g_{1}(\mathfrak{A}) I$, then it is clear that $r a_{1} g_{1}(b) \in g_{1}(\mathfrak{A})$, and so $g_{1}^{-1}\left(r a_{1}\right) b \in \mathfrak{A}$.

On the other hand, consider $\omega=a_{1} r \xi-\xi\left(g_{1}^{-1}\left(r a_{1}\right)\right)$. Since $g_{1}^{-1}(r) \in \mathfrak{A}$ and $a_{1} \in R$, it is clear that $\left(\xi\left(g_{1}^{-1}\left(r a_{1}\right)\right)\right) \cdot \mathfrak{A}=0$ and $\omega \cdot \mathfrak{A}=0$. It is easy to see that $\omega=\sum_{i=2}^{k}\left(a_{1} r a_{i}-a_{i}\left(\sigma_{i} \cdot\left(g_{1}^{-1}\left(r a_{1}\right)\right)\right)\right) \# x_{i}+\widetilde{y}$ for some $\widetilde{y} \in B \# C_{n-1}$. By the assumptions on $\mathbf{k}$ and $n, \omega$ cannot be contained in $D$, so $\omega \cdot B=0$. However, $\left(\xi\left(g_{1}^{-1}\left(r a_{1}\right)\right)\right) \cdot b=\xi \cdot\left(g_{1}^{-1}\left(r a_{1}\right) b\right) \in \xi \cdot \mathfrak{A}=0$; hence $a_{1} r \xi \cdot b=0$ for all $r \in g_{1}(\mathfrak{A}) I$. It follows that $a_{1} g_{1}(\mathfrak{A}) I(\xi \cdot b)=0$ and $g_{1}^{-1}\left(a_{1}\right) \mathfrak{A} g_{1}^{-1}(I(\xi \cdot b))=0$. We note that $g_{1}^{-1}\left(a_{1}\right) \mathfrak{A} \neq 0$, as $\ell(\mathfrak{A})=0$. Since $g_{1}^{-1}\left(a_{1}\right) \mathfrak{A}$ is a nonzero right ideal of a prime ring, we have $g_{1}^{-1}(I(\xi \cdot b))=0$, which implies the contradiction $\xi \cdot b=0$. Thus, we have the conclusion of the theorem. 
For a Hopf algebra $H$, we say that $R$ is a left $H$-module algebra if (1) $R$ is a left $H$-module and (2) $H$ measures $R$. In this case, the group-like elements of $H$ act as automorphisms of $R$. If $H$ is pointed, the action can be extended uniquely to the action on $R_{\mathcal{F}}$ ([M1, Corollary 3.5(2)]) and we can define a smash product algebra $R_{\mathcal{F}} \# H$ ([M2, 4.1.3]). As a corollary of Theorem 2, we have the following.

Corollary 3. Let $R$ be a prime algebra and $H$ a pointed Hopf algebra acting on $R$. If $\xi \cdot \mathfrak{A}=0$ for $\xi \in R_{\mathcal{F}} \# H$ and a right ideal $\mathfrak{A}$ of $R$ with $\ell(\mathfrak{A})=0$, then $\xi \cdot R_{\mathcal{F}}=0$.

\section{Automorphic-SKeW-DifFEREnTial Endomorphisms ON SEMIPRIME RINGS}

For any ring $A$, the set of all automorphisms of $A$ is denoted by $\operatorname{Aut}(A)$. For $\sigma \in \operatorname{Aut}(A)$, an additive map $d: A \rightarrow A$ is called a $\sigma$-derivation if $d(a b)=$ $d(a) b+\sigma(a) d(b)$ for all $a, b \in A$. The set of all $\sigma$-derivations is denoted by $\operatorname{Der}_{\sigma}(A)$. An element of $\bigcup_{\sigma \in \operatorname{Aut}(A)} \operatorname{Der}_{\sigma}(A)$ is called a skew-derivation.

Proposition 4. (1) Any automorphism $\sigma: R \rightarrow R$ can be extended to an automorphism of $R_{\mathcal{F}}$, and in this case, $\sigma(Q)=Q$.

(2) Any $\sigma$-derivation $d: R \rightarrow R$ can be extended to a $\sigma$-derivation of $R_{\mathcal{F}}$, and in this case, $d(Q) \subseteq Q$.

Proof. This is similar to [KP, Lemma 1].

According to Proposition 4, we can consider $\operatorname{Aut}(R) \subseteq \operatorname{Aut}\left(R_{\mathcal{F}}\right)$ and $\operatorname{Der}_{\sigma}(R) \subseteq$ $\operatorname{Der}_{\sigma}\left(R_{\mathcal{F}}\right)$ for $\sigma \in \operatorname{Aut}(R)$.

We regard $\operatorname{End}\left(R_{\mathcal{F}},+\right)$ as a $K$-module via $(c f)(x)=c(f(x))$ for $f \in \operatorname{End}\left(R_{\mathcal{F}},+\right)$, $c \in K$ and $x \in R_{\mathcal{F}}$.

Let $\mathcal{B}$ be a subring of $\operatorname{End}\left(R_{\mathcal{F}}\right)$ generated by $\left\{T_{a}, L_{a} \mid a \in R_{\mathcal{F}}\right\}$, Aut $(R)$ and $\cup \operatorname{Der}_{\sigma}(R)$. We call an element of $\mathcal{B}$ an automorphic-skew-differential endo$\sigma \in \operatorname{Aut}(R)$

morphism.

Before proving the semiprime case, we apply Theorem 2 to the automorphicskew-differential endomorphisms of prime rings.

For $f \in \mathcal{B}$, let $\left\{d_{i j} \in \operatorname{Der}_{\sigma_{i}}(R) \mid 1 \leq i \leq m, 1 \leq j \leq n_{i}\right\}$ be the set of all skew derivations and $\left\{g_{k}\right\}$ that of all automorphisms appearing in $f$. Removing the overlapping elements, we assume that $\left\{\sigma_{i}, g_{k} \mid 1 \leq i \leq m, 1 \leq k \leq t\right\}$ is the set of distinct automorphisms. Let $B$ be the algebra with generators $\left\{T_{i j}, X_{i}, Y_{k} \mid 1 \leq i \leq\right.$ $\left.m, 1 \leq j \leq n_{i}, 1 \leq k \leq t\right\}$. Then $B$ will be a pointed bialgebra under the following operations:

$$
\begin{gathered}
\Delta\left(T_{i j}\right)=T_{i j} \otimes 1+X_{i} \otimes T_{i j}, \Delta\left(X_{i}\right)=X_{i} \otimes X_{i}, \Delta\left(Y_{k}\right)=Y_{k} \otimes Y_{k}, \\
\varepsilon\left(T_{i j}\right)=0, \varepsilon\left(X_{i}\right)=\varepsilon\left(Y_{k}\right)=1 .
\end{gathered}
$$

We define the action of $B$ on $R_{\mathcal{F}}$ by $T_{i j} \cdot x=d_{i j}(x), X_{i} \cdot x=\sigma_{i}(x)$ and $Y_{k} \cdot x=$ $g_{k}(x)$ for $x \in R_{\mathcal{F}}$. We note that $R$ is $B$-stable.

Now, we have an element $\xi \in R_{\mathcal{F}} \# B$ corresponding to $f$. As $\xi \cdot r=f(r)$ for any $r \in R$ and $B$ is pointed, we have the following result as an easy consequence of Theorem 2 .

Theorem 5. Let $R$ be a prime ring and $f$ an automorphic-skew-differential endomorphism. If $f(\mathfrak{A})=0$ for some right ideal $\mathfrak{A}$ with $\ell(\mathfrak{A})=0$, then $f(R)=0$. 
The next theorem shows that Theorem 5 holds even if $R$ is a semiprime ring. It is the main result of this section.

Theorem 6. Let $R$ be a semiprime ring and $f$ an automorphic-skew-differential endomorphism. If $f(\mathfrak{A})=0$ for some right ideal $\mathfrak{A}$ with $\ell(\mathfrak{A})=0$, then $f(R)=0$.

We need several lemmas to prove this theorem. For the remainder of this note, we assume that $R$ is a semiprime ring. The set of all idempotents of $K$ is represented by $E$. Let $e_{1}, e_{2}$ be elements in $E$. The operation $\oplus$ defined by $e_{1} \oplus e_{2}=e_{1}+e_{2}-2 e_{1} e_{2}$ and the ordinary multiplication make $E$ into a Boolean ring. Besides, we define the partial order $\leq$ of $E$ by $e_{1} \leq e_{2} \Leftrightarrow e_{1} e_{2}=e_{1}$. When $E_{0}$ is a nonempty subset of $E$, a nonzero element $e \in E_{0}$ is called minimal in $E_{0}$ if $e^{\prime} \leq e$ for $0 \neq e^{\prime} \in E_{0}$ implies $e^{\prime}=e$.

A finite subset of $E$ generates a finite Boolean subring.

For every nonempty subset $S$ of $R_{\mathcal{F}}$, we can uniquely determine an idempotent $e=e(S) \in E$ satisfying (i) $e s=s$ for all $s \in S$ and (ii) $e x=0$ for any $x \in R_{\mathcal{F}}$ such that $S R x=0([\mathrm{~K} 2, \mathrm{p} .26])$.

For $\sigma \in \operatorname{Aut}(R)$ and $q \in Q$, set $\left(a d_{\sigma} q\right)(x)=q x-\sigma(x) q$. Then $a d_{\sigma} q \in \operatorname{Der}_{\sigma}\left(R_{\mathcal{F}}\right)$.

Proposition 7 ([K1, Lemma 10]). Any $d \in \operatorname{Der}_{\sigma}(R)$ can be uniquely decomposed into the sum $a d_{\sigma} q+\mu_{\sigma}$ for some $q \in Q$ and some left and right E-linear $\sigma$-derivation $\mu_{\sigma}$.

For any $x \in R, a \in R_{\mathcal{F}}, g, \sigma \in \operatorname{Aut}(R)$ and $\sigma$-derivation $d$, we have the following relations.

(1) $d L_{a}(x)=d(a x)=d(a) x+\sigma(a) d(x)=\left(L_{d(a)}+L_{\sigma(a)} d\right)(x)$.

(2) $d T_{a}(x)=d(x a)=d(x) a+\sigma(x) d(a)=\left(T_{a} d+T_{d(a)} \sigma\right)(x)$.

(3) $g L_{a}(x)=g(a x)=g(a) g(x)=L_{g(a)} g(x)$.

(4) $g T_{a}(x)=g(x a)=g(x) g(a)=T_{g(a)} g(x)$.

(5) $a d_{\sigma} a(x)=a x-\sigma(x) a=\left(L_{a}-T_{a} \sigma\right)(x)$.

(6) $d g(x)=g g^{-1} d g(x)$.

It is easy to see that $g^{-1} d g$ is a skew-derivation in $\operatorname{Der}_{g^{-1} \sigma g}(R)$. Using the decomposition in Proposition 7, we may assume that an automorphic-skew-differential endomorphism is the sum of monomials of the form $T_{a} L_{b} g \Delta$ for $a, b \in R_{\mathcal{F}}, g \in \operatorname{Aut}(R)$ and a composition map of $E$-linear skew-derivations $\Delta$.

Proposition 8 ([K2, p. 110]). Let $f$ be an automorphic-skew-differential endomorphism of $R$ such that $f(\mathfrak{A})=0$ for some right ideal of $R$ with $\ell(\mathfrak{A})=0$. For any $e \in E$, we have an idempotent $0 \neq e^{\prime} \leq e$ such that $e^{\prime} f$ can be decomposed into the sum $\sum_{\lambda} f_{\lambda}$ satisfying (1) $f_{\lambda}\left(\mathfrak{A}_{\lambda}\right)=0$ for some $\mathfrak{A}_{\lambda} \triangleleft_{r} R$ with $\ell\left(\mathfrak{A}_{\lambda}\right)=0$ and (2) for any idempotent $e^{\prime \prime} \leq e^{\prime}$ and automorphism $g$ appearing in $f_{\lambda}, g\left(e^{\prime \prime}\right)=g_{\lambda}\left(e^{\prime \prime}\right)$ for some $g_{\lambda} \in \operatorname{Aut}(R)$.

Proof. Let $\left\{g_{\mu} \mid \mu \in M\right\}$ be the set of all distinct automorphisms appearing in $f$.

If $g_{\mu}\left(e_{1}\right)=g_{\nu}\left(e_{1}\right)$ for any $\mu, \nu \in M$ and idempotent $e_{1} \leq e$, we have the conclusion on setting $e^{\prime}=e$. We assume that for some $e_{1} \leq e$ and $\mu, \nu \in M$ we have $g_{\mu}\left(e_{1}\right) \neq g_{\nu}\left(e_{1}\right)$. Let $M^{\prime}$ be a subset of $M$ so that $\left\{g_{\mu}\left(e_{1}\right) \mid \mu \in M^{\prime}\right\}$ is the set of all distinct idempotents in $\left\{g_{\mu}\left(e_{1}\right) \mid \mu \in M\right\}$. Then $f\left(x e_{1}\right)=\sum_{\mu \in M^{\prime}} f_{\mu}(x) g_{\mu}\left(e_{1}\right)$ for some $f_{\mu} \in \mathcal{B}$ with $f=\sum_{\mu} f_{\mu}$. Let $e_{2}$ be a minimal idempotent in the Boolean ring gen- 
erated by $\left\{g_{\mu}\left(e_{1}\right)\right\}$ satisfying $\left\{\mu \in M^{\prime} \mid e_{2} g_{\mu}\left(e_{1}\right) \neq 0\right\} \neq \emptyset$ and $\left\{\mu \in M^{\prime} \mid e_{2} g_{\mu}\left(e_{1}\right)=\right.$ $0\} \neq \emptyset$. Set $f_{1}(x)=f(x) e_{2}-f\left(x e_{1}\right) e_{2}$ and $f_{2}(x)=f\left(x e_{1}\right) e_{2}$. Then $e_{2} f=f_{1}+f_{2}$ and $f_{2} \neq 0$. Moreover, for $I \in \mathcal{F}$ with $I e_{1} \subseteq R$, we have $f_{1}(\mathfrak{A} I)=f_{2}(\mathfrak{A} I)=0$, as $\mathfrak{A} I e_{1} \subseteq \mathfrak{A}$. It is easy to see that $\ell(\mathfrak{A} I)=0$. As each number of the distinct automorphisms appearing in $f_{1}$ and $f_{2}$ is less than that of $f$, continuing this process, we get the conclusion of the proposition.

Proposition 9. Let $f$ be an automorphic-skew-differential endomorphism of $R$. Assume that for any $e \in E$, there exists $e^{\prime} \in E$ satisfying $0 \neq e^{\prime} \leq e$ and $f(R) e^{\prime}=0$. Then we have $f(R)=0$.

Proof. See the proof of [K2, Theorem 2.3.6 (p.109)].

Here, we write $e^{\prime} f=\sum f_{\lambda}$ as shown in Proposition 8. If we can show that $f_{\lambda}(R)=0$ for each $f_{\lambda}$, it follows that $e^{\prime} f(R)=0$, and by Proposition 9 we obtain the conclusion of Theorem 6. Hence, we may assume that $g_{\lambda}\left(e^{\prime}\right)=e^{\prime}$, as it is sufficient to show that $g_{\lambda}^{-1}\left(f_{\lambda}(R)\right)=0$ for each $\lambda$. So, we assume that $f_{\lambda}\left(e^{\prime \prime} x\right)=e^{\prime \prime} f_{\lambda}(x)$ for any idempotent $e^{\prime \prime} \leq e^{\prime}$. In this case, $f_{\lambda}(x)=e^{\prime} f_{\lambda}(x)=f_{\lambda}\left(e^{\prime} x\right)$, and thus our aim is to show that $f_{\lambda}\left(e^{\prime} R\right)=0$ for a semiprime ring $e^{\prime} R$. From the above argument, setting $e^{\prime}=1$ and $f=f_{\lambda}$, we may assume every automorphism appearing in $f$ is E-linear.

According to [K2, p.59], we induce a certain "topology" on the $K$-module $M$. Let $\Gamma$ be a directed set. We call $m \in M$ a limit of a family $\left\{m_{\alpha} \in M \mid \alpha \in \Gamma\right\}$ if there exists a family $\left\{e_{\alpha} \in E \mid \alpha \in \Gamma\right\}$ satisfying

(i) $e_{\alpha} \leq e_{\beta}$ if $\alpha \leq \beta$

(ii) $\sup \left\{e_{\alpha} \mid \alpha \in \Gamma\right\}=1$, and

(iii) $m e_{\alpha}=m_{\alpha} e_{\alpha}$ for all $\alpha \in \Gamma$.

A subset $S \subseteq M$ is said to be closed if $S$ contains any limit of the family in $S$. The least closed set containing $T \subseteq M$ is called the closure of $T$ and denoted by $\widehat{T}$. For $K$-modules $M_{1}, M_{2}$, a mapping $\varphi: M_{1} \rightarrow M_{2}$ is called completely continuous if $\varphi(m)=\lim _{\alpha \in \Gamma} \varphi\left(m_{\alpha}\right)$ for any $m, m_{\alpha} \in M_{1}(\alpha \in \Gamma)$ with $m=\lim _{\alpha \in \Gamma} m_{\alpha}$. All automorphisms and $E$-linear skew-derivations are completely continuous.

Lemma 10 ([K2, Lemma 1.6.6, 1.6.10, 1.6.11, 1.6.26]). (1) If $f(T)=0$ for an automorphic-skew-differential endomorphism $f$ and $T \subseteq R_{\mathcal{F}}$, then $f(\widehat{T})=0$.

(2) If $T$ is a subring of $R_{\mathcal{F}}$, so is $\widehat{T}$.

(3) If $I$ is a right ideal of a subring $T, \widehat{I}$ is a right ideal of $\widehat{T}$.

(4) Let $T$ be a closed additive subset of $R_{\mathcal{F}}$ with $T E \subseteq T$ and $f: T \rightarrow R_{\mathcal{F}}$ an E-linear mapping. There is an element $t \in T$ satisfying $e(f(t))=e(f(T))$.

By Lemma $10(2), \widehat{R E}$ is a subring of $Q$, where $R E$ is the set of all linear combinations $\sum a_{i} e_{i}, a_{i} \in R, e_{i} \in E$. Let $\wp$ be a maximal ideal of $K$. The ideal of $R_{\mathcal{F}}$ generated by $\wp$ is denoted by $\wp R_{\mathcal{F}}$. Let $\phi$ be a canonical homomorphism $R_{\mathcal{F}} \rightarrow R_{\mathcal{F}} / \wp R_{\mathcal{F}}$. Then $\phi(\widehat{R E})$ is a prime ring ([K2, Lemma 1.12.1]). Moreover, $\phi\left(R_{\mathcal{F}}\right)$ is contained in the left Martindale quotient ring of $\phi(\widehat{R E})$ ([K2, Proposition 1.12.3]). We denote the left Martindale quotient ring by $\phi(\widehat{R E})_{\mathcal{F}}$.

Lemma 11. For $a \in R_{\mathcal{F}}, a \in \operatorname{ker} \phi \Leftrightarrow e(a) \in \wp$.

Proof. See the proof of [K2, Lemma 1.9.18]. 
Lemma 12. Let $\mathfrak{A}$ be a right ideal of $R$ with $\ell(\mathfrak{A})=0$, and a an element of $R_{\mathcal{F}}$. Then $a \widehat{\mathfrak{A E}} \subseteq \operatorname{ker} \phi \Leftrightarrow a \in \operatorname{ker} \phi$.

Proof. Assume that $a \widehat{\mathcal{A} E} \subseteq \operatorname{ker} \phi$. By Lemma 10(4), there exists $t \in \widehat{\mathscr{A} E}$ with $e(a t)=e(a \widehat{\mathfrak{A} E})$. As $(1-e(a t)) a \widehat{\mathfrak{A} E}=0$ and $\ell_{\widehat{R E}}(\widehat{\mathfrak{A} E})=0$ for $\ell(\mathfrak{A})=0$, we have $(1-e(a t)) a=0$, and so $e(a)=e(a) e(a t)$. Since $a t \in \operatorname{ker} \phi$, by Lemma 11, $e(a t) \in \wp$. Thus, $e(a) \in \wp$ and $a \in \operatorname{ker} \phi$. The other direction is easy.

From Lemma 11, it is clear that $\operatorname{ker} \phi \cap Q=E(\wp) Q$, where $E(\wp)=\wp \cap E$. So this ideal is invariant under any $E$-linear mapping $\beta: Q \rightarrow Q$. Thus, we can define a mapping $\widehat{\beta}$ of $\phi(Q)$ by $\widehat{\beta}(\phi(q))=\phi(\beta(q))$.

Moreover, if $\beta$ is an automorphism or skew-derivation, by restricting $\widehat{\beta}$ to $\phi(\widehat{R E})$, we have an automorphism or skew-derivation of $\phi(\widehat{R E})_{\mathcal{F}}$ from Proposition 4 . We denote it by the same $\widehat{\beta}$.

Now, as all automorphisms and skew-derivations appearing in $f$ are $E$-linear, we can define $\widehat{f}$, which is an automorphic-skew-differential endomorphism of a prime ring $\phi(\widehat{R E})$, by changing each automorphism $g$ to $\widehat{g}$, each skew-derivation $d$ to $\widehat{d}$ and coefficient $a$ to $\phi(a) \in \phi(\widehat{R E})_{\mathcal{F}}$.

Proof of Theorem 6. Let $\wp$ and $\phi$ be as above. For $\mathfrak{A} \triangleleft_{r} R$ with $f(\mathfrak{A})=0$ and $\ell(\mathfrak{A})=0, \phi(\widehat{\mathfrak{A} E})$ is a right ideal of $\phi(\widehat{R E})$ and $\widehat{f}(\phi(\widehat{\mathfrak{A} E}))=\phi(f(\widehat{\mathfrak{A E}}))=0$ by the fact that $f$ is $E$-linear and Lemma 10(1),(3). By Lemma $12, \ell_{\phi(\widehat{R E})}(\phi(\widehat{\mathfrak{A E}}))=0$, so we can apply Theorem 5 to a prime ring $\phi(\widehat{R E})$ and $\widehat{f}$; we have $\widehat{f}(\phi(\widehat{R E}))=0$. Thus, $f(R) \subseteq f(\widehat{R E}) \subseteq \wp R_{\mathcal{F}}$ for each maximal ideal $\wp$ of $K$.

Assume that $a$ is contained in $\wp R_{\mathcal{F}}$ for any maximal ideal $\wp \triangleleft K$. By Lemma $11, e(a)$ is contained in $\bigcap_{\wp} E(\wp) \subset \bigcap_{\wp} \wp$, where $\wp$ runs over all maximal ideals of $K$. Since $K$ is a regular ring, the intersection of all maximal ideals is zero. Hence $a=e(a) a=0$.

Thus, we have $f(R)=0$, and the proof is completed.

\section{ACKNOWLEDGEMENTS}

The author thanks the referee for helpful comments, in particular many detailed suggestions for Theorem 2 .

\section{REFERENCES}

[B] J. Bergen, Automorphic-differential identities in rings, Proc. Amer. Math. Soc. 106 (1989), 297-305. MR 89k:16064

[K1] V. K. Kharchenko, Skew derivations of semiprime rings, Siberian Math. J. 32 (1991), 1045-1051. MR 93b:16032

[K2] V. K. Kharchenko, Automorphisms and derivations of associative rings, Kluwer, Dordrecht, 1991. MR 93i: 16048

[KP] V. K. Kharchenko and A. Z. Popov, Skew derivations of prime rings, Comm. Algebra 20 (1992), 3321-3345. MR 93k:16071

[Ma] T. Marlowe, The diagonal of a pointed coalgebra and incidence-like structure, J. Pure and Appl. Algebra 35 (1985), 157-169. MR 86j:16002

[M1] S. Montgomery, Bi-invertible actions of Hopf algebras, Isr. J. Math. 83 (1993), 45-71. MR 94g: 16047

[M2] S. Montgomery, Hopf algebras and their actions on rings, CBMS Regional Conference Series in Mathematics 82, AMS, Providence, R.I., 1993. MR 94i:16019 
[O] A. Ouarit, Identités auto-différentielles d'anneaux semi-primiers, C. R. Acad. Sci. Paris 314 (1992), 173-176. MR 93c:16026

[TW] E. Taft and R. Wilson, On antipodes in pointed Hopf algebras, J. Algebra 29 (1974), 27-32. MR 49:2820

Department of Mathematics, Nimhama National College of Technology, 7-1 YagumoCho, Ninhama, Ehime, 792, Japan

E-mail address: yanai@sci.niihama-nct.ac.jp 